\title{
Color vision abnormality as an initial presentation of the complete type of congenital stationary night blindness
}

This article was published in the following Dove Press journal:

Clinical Ophthalmology

6 August 2013

Number of times this article has been viewed

Xue Tan

Aya Aoki

Yasuo Yanagi

Department of Ophthalmology, University of Tokyo School of Medicine, Hongo, Bunkyo-ku, Tokyo, Japan
Correspondence: Yasuo Yanagi Department of Ophthalmology, University of Tokyo School of Medicine, 7-3-I Hongo, Bunkyo-ku, Tokyo I I 3-8655, Japan

Tel +8I 358008660

Fax +8I 338170798

Email yanagi-tky@umin.ac.jp
Abstract: Patients with the complete form of congenital stationary night blindness (CSNB) often have reduced visual acuity, myopia, impaired night vision, and sometimes nystagmus and strabismus, however, they seldom complain of color vision abnormality. A 17-year-old male who was at technical school showed abnormalities in the color perception test for employment, and was referred to our hospital for a detailed examination. He had no family history of color vision deficiency and no other symptoms. During the initial examination, his best-corrected visual acuity was 1.2 in both eyes. His fundus showed no abnormalities except for somewhat yellowish reflex in the fovea of both eyes. Electroretinogram (ERG) showed a good response in cone ERG and $30 \mathrm{~Hz}$ flicker ERG, however, the bright flash, mixed rod and cone ERG showed a negative type with a reduced b-wave (positive deflection). There was no response in the rod ERG, either. From the findings of the typical ERG, the patient was diagnosed with complete congenital stationary night blindness. This case underscores the importance of ERG in order to diagnose the cause of a color vision anomaly.

Keywords: congenital stationary night blindness, CSNB, electroretinogram, ERG, color vision defect

\section{Introduction}

Congenital stationary night blindness (CSNB; CSNB1A, OMIM \#310500, CSNB1B, OMIM\#257270, CSNB1C, OMIM\#613216 and CSNB2A, OMIM\#3000071, CSNB 2B, OMIM \#610427) (Online Mendelian inheritance in men) is a clinically heterogeneous group of nonprogressive retinal disorders characterized by impaired night vision, decreased visual acuity, nystagmus, myopia, and strabismus. ${ }^{1}$ A negative-type electroretinogram (ERG) recording, with a normal a-wave (initial negative deflection) and significantly reduced b-wave (positive deflection) amplitude, is recorded in the bright flash, mixed rod and cone ERG in CSNB. CSNB can be classified into complete and incomplete forms based on the ERG. Complete CSNB (CSNB1) is characterized by reduced rod b-wave response due to a complete defect of the metabotropic (ON)-bipolar cell-mediated signal transduction, whereas incomplete CSNB (CSNB2) is characterized by both a reduced rod b-wave and substantially reduced flicker cone responses due to the dysfunction of both $\mathrm{ON}$ and ionotropic (OFF) bipolar cell-mediated signal transduction.

Complete CSNB patients have normal fundi; they have malfunction in neurotransmission from rods to bipolar cells. ${ }^{1,2}$ The hereditary mode of complete CSNB is either autosomal recessive or X-linked recessive. Complete CSNB is caused by mutations in the genes $N Y X$, GRM6, and TRPM1.,4 Typical clinical features of complete CSNB consist of night blindness from early childhood and normal to mildly reduced 
best-corrected visual acuities and high or moderate myopia with astigmatism. In recent research, it is reported that none of the CSNB1 patients had severe abnormal color defects, but $14 \%$ had mild color defects. ${ }^{5}$ Signals from the blue cones are mainly transferred through the ON-bipolar pathway, but CSNB1 patients have no function in this pathway. ${ }^{1,6}$ The reason why CSNB1 patients usually do not show color vision abnormalities is unclear. In the current report, we document the clinical characteristics of a patient with complete CSNB who was asymptomatic and referred for the investigation of the cause of a color vision defects. The current report underscores the importance of electrophysiological examination in the diagnosis of CSNB.

\section{Case report}

A 17-year-old male who was in a technical school of the transportation industry showed abnormalities in the color perception test for employment, and was referred to our hospital for a detailed examination. While he was in the elementary school, he received a color perception test during a health check. There were no abnormalities on the examination. He had no family history of color vision deficiency, and had no other symptoms. During the initial examination, his best-corrected visual acuity was 1.2 , with no refractive error in either eye. The referral doctor, who specialized in color vision, investigated his color vision. Testing with Ishihara pseudoisochromatic plates, the patient could read only the first plate, and was diagnosed to have a color vision defect. However, he passed the panel D-15 dichotomous test with no errors. The patient's color vision defect was mild, with no definite axis of error according to the 100-hue test performed at the referral hospital, and he showed normal match with an anomaloscope color blindness test.

The referring doctor also performed Standard Pseudoisochromatic Plates, part 2 to assess the type of color defect. The patient made two mistakes for blue-yellow differentiation and five mistakes for red-green differentiation in the right eye; and two mistakes for blue-yellow differentiation and four mistakes for red-green differentiation in the left eye, suggesting that the color vision defect had no definite axis.

During the initial examination, his fundus showed no abnormalities except for a somewhat yellowish reflex in the fovea of both eyes. Fundus autofluorescence, optical coherence tomography, and multifocal ERG were normal (Figure 1). Fluorescein fundus angiography also revealed no abnormalities (Figure 1). Color vision deficiency with no apparent retinal abnormalities is sometimes associated with retinal diseases. ${ }^{7}$ Thus, these findings prompted us to
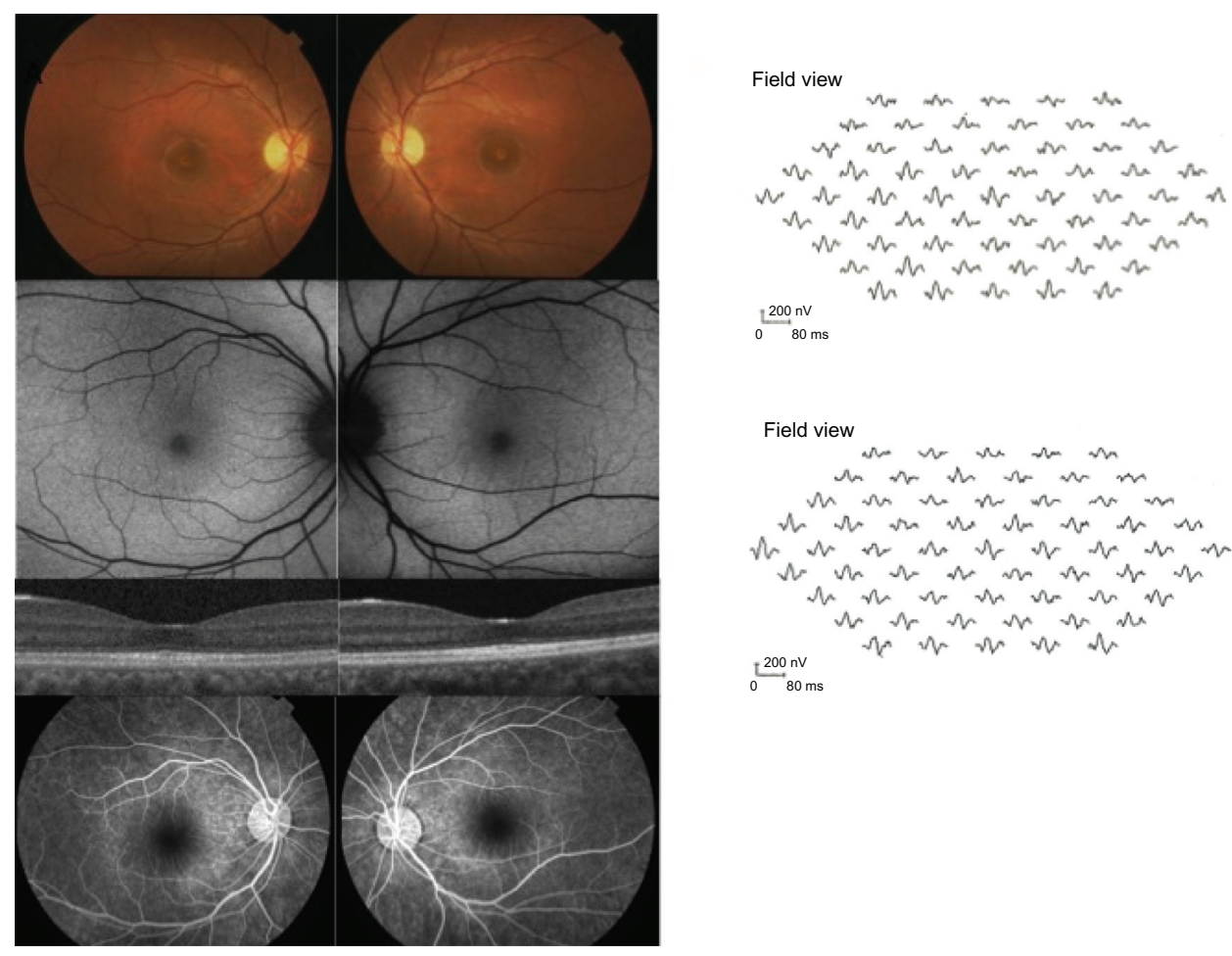

Field view

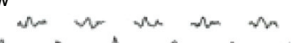

Figure I Fundus photograph of the patient.

Notes: Fundus photograph shows normal fundus except for a somewhat yellowish reflex in the macula of both eyes. Fundus autofluorescence, optical coherence tomography, and multifocal ERG are normal. Fluorescein angiography also revealed no abnormalities.

Abbreviation: ERG, electroretinogram. 
obtain an ERG. Both the cone ERG and $30 \mathrm{~Hz}$ flicker ERG (recorded by International Society for Clinical Electrophysiology Vision [ISCEV] standard protocol ERG [using Tomei LE3000, Tomei, Tokyo, Japan]) showed a good response; however, the cone ERG showed wide a-wave trough. ${ }^{7}$ Bright flash, mixed rod and cone ERG showed a negative type with a reduced $b$-wave and a disappearance of oscillatory potential. No response was recorded in the rod ERG, either (Figure 2). Based on the ERG findings, the patient was diagnosed with complete CSNB. Observation was recommended; he has

Patient

Normal

Rod

Rod
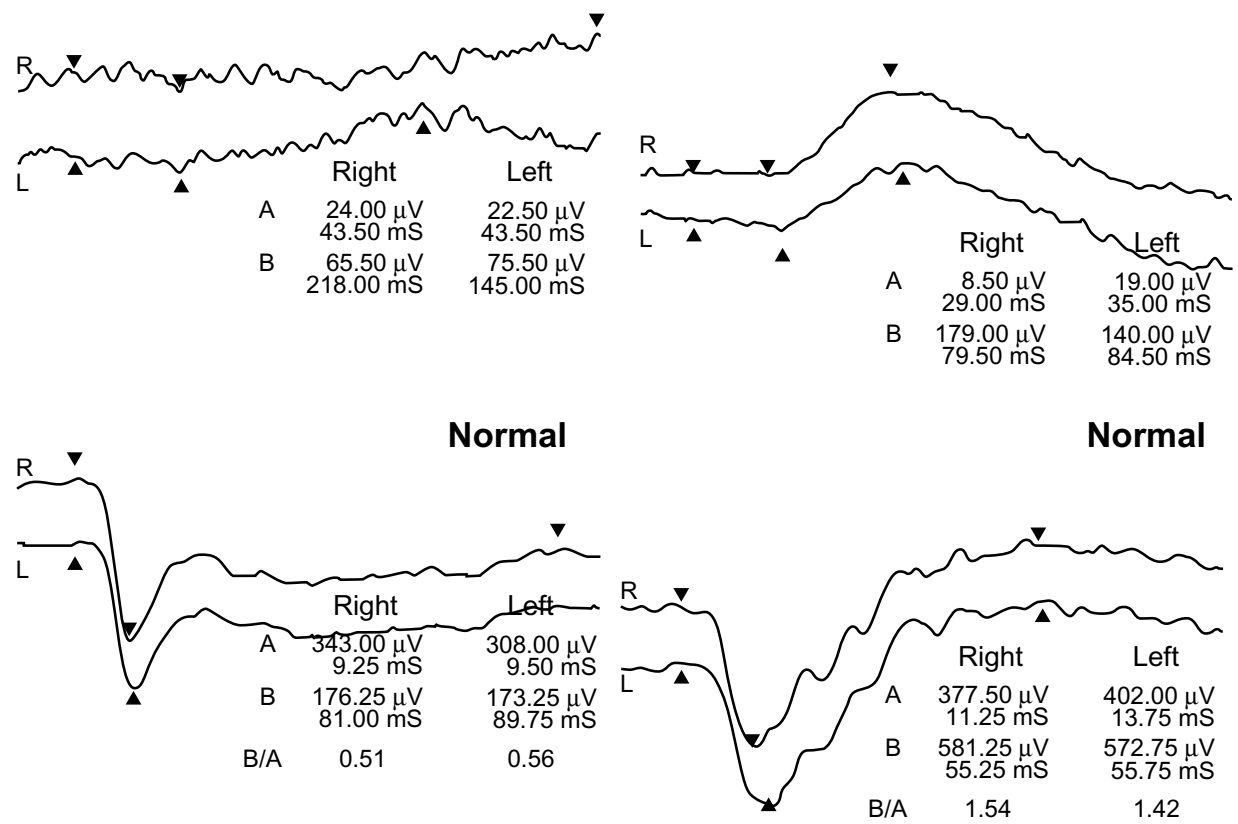

Cone

Cone

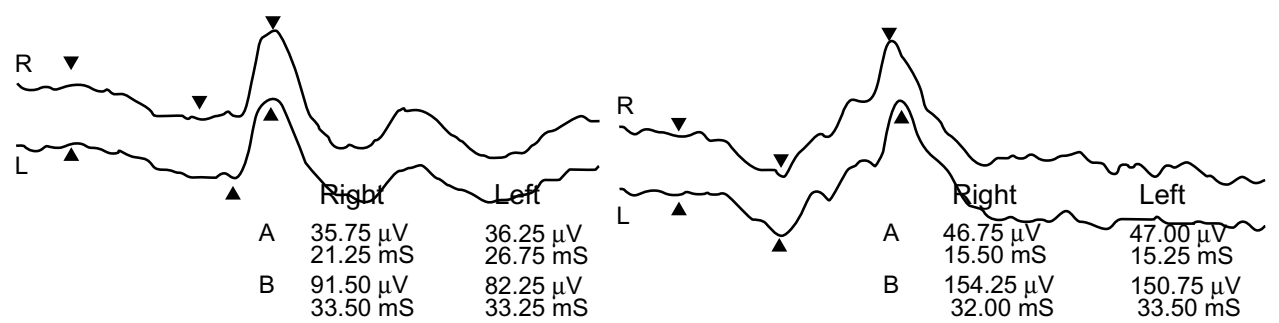

$30 \mathrm{~Hz}$ flicker

$30 \mathrm{~Hz}$ flicker

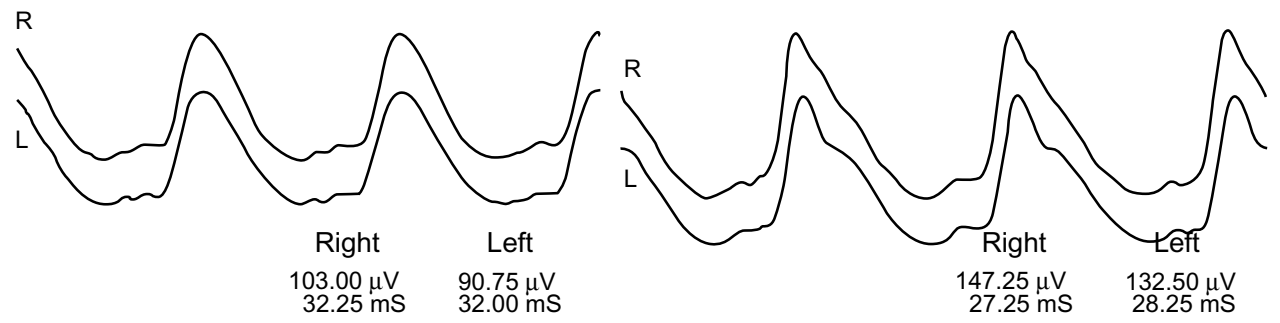

Figure 2 Recording of ERG from the patient.

Notes: Whereas both the cone ERG and $30 \mathrm{~Hz}$ flicker ERG shows a good response, the bright-flash ERG shows a negative type with a reduced b-wave amplitude and a disappearance of oscillatory potential. In addition, the rod ERG shows no rod response.

Abbreviation: ERG, electroretinogram. 
been monitored for 5 years, and remains asymptomatic with no changes in visual acuity and fundus appearance since the first visit.

\section{Discussion}

There are two forms of CSNB: complete and incomplete. It has been recognized that complete and incomplete CSNB are different diseases, with different ERG findings and different gene mutations. ${ }^{1,2}$ Complete CSNB patients show a negative type with reduced b-wave amplitude in the mixed rod and cone ERG and the disappearance of rod response in the scotopic ERG, whereas incomplete CSNB patients have residual rod function but cone dysfunction. In complete CSNB, the bright flash cone response presents a characteristic wide a-wave trough and moderate reduction of the b-wave amplitude. ${ }^{8}$ Complete CSNB is either autosomal recessive or X-linked recessive, whereas incomplete CSNB is usually $\mathrm{X}$-linked recessive. ${ }^{2}$ Most of the causative gene products in complete CSNB (ie, NYX, GRM6 and TRPM1, and GPR179) are localized at the dendritic terminals of ON bipolar cells and contribute the cell activity. ${ }^{3,5}$ There are also differences in other clinical findings; patients complain of low visual acuity in both types, but complete CSNB patients present with moderate or high myopia, whereas incomplete CSNB patients present with either myopia or hyperopia. ${ }^{1}$ Sometimes strabismus and nystagmus are also found. In a previous report, $20 \%$ to $40 \%$ of patients with CSNB were found to present with nystagmus. ${ }^{9}$ There is little complaint of night blindness in incomplete CSNB, which is in contrast to patients with complete CSNB, who usually complain of night blindness. ${ }^{5}$ In the recent literature, all patients with complete CSNB or CSNB1 reported night vision problems. Moreover, the clinical features of patients with complete CSNB are reported to be similar, though the causative gene is different. ${ }^{3,5}$ This is in contrast with incomplete CSNB, which is reported to show considerable variability in the clinical expression.

The current case is a rare presentation of a patient with complete CSNB. Although CSNB is characterized by impaired night vision and/or decreased visual acuity, myopia, and nystagmus, the patient had neither congenital color blindness nor other symptoms; he merely happened to

Clinical Ophthalmology

\section{Publish your work in this journal}

Clinical Ophthalmology is an international, peer-reviewed journal covering all subspecialties within ophthalmology. Key topics include: Optometry; Visual science; Pharmacology and drug therapy in eye diseases; Basic Sciences; Primary and Secondary eye care; Patient Safety and Quality of Care Improvements. This journal is indexed on Submit your manuscript here: http://www.dovepress.com/clinical-ophthalmology-journal present a color vision anomaly in screening tests. Although the results of the color vision defect were moderate and nonspecific, they led us to suspect retinal disorder. The ophthalmological examination revealed atypical findings; however, the patient has been diagnosed with complete CSNB by the characteristic findings of the ERG. This case underscores the importance of careful patient examination and ERG in order to diagnose the cause of a color vision anomaly. Clinicians should be more aware of retinal disease with normal fundus and normal visual acuity. ${ }^{5}$ As a recent study reports, patients with good visual acuity may not always be recognized as having a retinal disorder. ${ }^{5}$ The yellowish reflex of the fovea in the current case is likely to reflect the increased macular xanthophylls, however, the association of the foveal xanthophylls and complete CSNB remains to be clarified in future studies.

\section{Disclosure}

The authors report no conflicts of interest in this work.

\section{References}

1. Miyake Y, Yagasaki K, Horiguchi M, Kawase Y, Kanda T. Congenital stationary night blindness with negative electroretinogram. A new classification. Arch Ophthalmol. 1986;104(7):1013-1020.

2. Miyake Y. Establishment of the concept of new clinical entities complete and incomplete form of congenital stationary night blindness. Nippon Ganka Gakkai Zasshi. 2002;106(12):737-755. Japanese.

3. Nakamura M, Sanuki R, Yasuma TR, et al. TRPM1 mutations are associated with the complete form of congenital stationary night blindness. Mol Vis. 2010;16:425-437.

4. Naeem MA, Chavali VR, Ali S, et al. GNAT1 associated with autosomal recessive congenital stationary night blindness. Invest Ophthalmol Vis Sci. 2012;53(3):1353-1361.

5. Bijveld MM, Florijn RJ, Bergen AA, et al. Genotype and phenotype of 101 Dutch patients with congenital stationary night blindness. Ophthalmology. E-pub 2013.

6. Kamiyama M, Yamamoto S, Nitta K, Hayasaka S. Undetectable S cone electroretinogram b-wave in complete congenital stationary night blindness. Br J Ophthalmol. 1996;80(7):637-639.

7. Weleber RG, Gregory-Evans K. Retinis pigmentosa and allied disorders. In: Ryan S, editor. Retina. 4th ed. St Louis: Mosby; 2006: 395-498.

8. Lachapelle P, Little JM, Polomeno RC. The photopic electroretinogram in congenital stationary night blindness with myopia. Invest Ophthalmol Vis Sci. 1983;24(4):442-450.

9. Pieh C, Simonsz-Toth B, Gottlob I. Nystagmus characteristics in congenital stationary night blindness (CSNB). Br J Ophthalmol. 2008;92(2): 236-240.

PubMed Central and CAS, and is the official journal of The Society of Clinical Ophthalmology (SCO). The manuscript management system is completely online and includes a very quick and fair peer-review system, which is all easy to use. Visit http://www.dovepress.com/ testimonials.php to read real quotes from published authors. 